\title{
Low Carb Diet Outranked a Low Fat Diet in Weight Loss
}

\author{
Shabnam Ansari (ib ${ }^{1, *}$, Suhail Fatima ${ }^{2}$ and Nazeem Fahamiya ${ }^{3}$ \\ ${ }^{1}$ Department of Biotechnology, Faculty of Natural Sciences, Jamia Millia Islamia, New Delhi, India \\ ${ }^{2}$ Department of Amraze Niswan wa Qabalat, School of Unani Medical Education and Research, Jamia Hamdard, New Delhi, India \\ ${ }^{3}$ Department of Ilmul Advia, Institute of Indigenous Medicine, University of Colombo, Rajagiriya, Sri Lanka \\ "Corresponding author: Department of Biotechnology, Faculty of Natural Sciences, Jamia Millia Islamia, New Delhi, India. Email: drshabnamansari.md@gmail.com
}

Received 2019 July 22; Accepted 2019 August 18.

\section{Dear Editor,}

As per the study published in the Annals of Internal Medicine, 150 men and women of the racially diverse group were recruited to follow a specific diet for a period of one year, which particularly restricted them to eat wither carbohydrate or fat, but not overall calories.

At the end of the clinical trial, a group of people who have followed a low carbohydrate diet had lost about a mean of eight pounds more than a group of people have followed a low-fat diet. In addition, people in a low carbohydrate diet had shown a considerable drop in body fat percentage than people who were following the low-fat diet. People in the low-fat group also experienced weight loss but was the loss of more muscle mass than fat. The balance of lean mass versus fat mass is much more important than weight. Hence, the low-carbohydrate, high-fat group people showed more weight loss than the low-fat group.

Nonetheless, people who restrict carbohydrates tend to lose extra body fat and thereby, have a lesser risk of heart and cerebrovascular diseases than with a low-fat diet consumption, which healthcare community has advised to follow since years.

Recent dietary researches observed that the risk of heart disease may get reduced by eating fewer carbohydrates and moderate dietary fat except for trans fats. Nuts are favorable for health and heart as they contain less sugar and have healthier saturated fats, which favor more in weight loss because of their anti-oxidant properties. These novel findings infer that low carbohydrate diet consumption is extra efficacious in losing fat content and helps in the reduction of weight (1).

Another study was recently published in the JAMA/Archives journals (Archives of Internal Medicine). Researchers of St. Michael's Hospital and the University of Toronto, Ontario, Canada, evaluated the effectiveness of a low-carbohydrate diet on weight loss in 50 subjects. A total of 25 subjects were randomly allocated to consume "the "Eco-Atkins" diet" for a month or four weeks, while another 25 participants are allowed to eat a control diet of high-carbohydrate, Lacto-Ovo vegetarian, and based on low-fat dairy and whole-grain products.
The researchers observed the similar amount of weight loss in both groups, however, reductions in LDL-levels (bad cholesterol) and improvements in the ratios between total cholesterol and HDL-C (good cholesterol) were greater in the low-carbohydrate diet than a high-carbohydrate diet. The low-carbohydrate diet also showed improvement in levels and ratios of apolipoproteins and proteins, which bind to fatty acids. In addition, small but significant reductions were also observed in both systolic and diastolic blood pressure in the low-carbohydrate than the highcarbohydrate group.

Thus, these studies showed that obese or overweight people with high LDL cholesterol levels who consumed a diet, high in vegetable proteins have accomplished a greater reduction in cholesterol levels and weight, a culprit for heart diseases. These studies also infer that intake of a low-fat diet with high carbohydrate may also lead to weight loss but doesn't exclude the risk of heart disease (2).

\section{Footnotes}

Conflict of Interests: The authors certify that they have no affiliations with or involvement in any organization or entity with any financial interest (such as honoraria; educational grants; participation in speakers' bureaus; membership, employment, consultancies, stock ownership, or other equity interest; and expert testimony or patentlicensing arrangements), or non-financial interest (such as personal or professional relationships, affiliations, knowledge or beliefs) in the subject matter or materials discussed in this manuscript.

Funding/Support: There is no funding/support.

\section{References}

1. Bazzano LA, Hu T, Reynolds K, Yao L, Bunol C, Liu Y, et al. Effects of low-carbohydrate and low-fat diets: A randomized trial. Ann Intern Med. 2014,161(5):309-18. doi: 10.7326/M14-0180. [PubMed: 25178568]. [PubMed Central: PMC4428290].

2. Yancy WS Jr, Westman EC, McDuffie JR, Grambow SC, Jeffreys AS, Bolton $\mathrm{J}$, et al. A randomized trial of a low-carbohydrate diet vs orlistat plus a low-fat diet for weight loss. Arch Intern Med. 2010;170(2):136-45. doi: 10.1001/archinternmed.2009.492. [PubMed: 20101008]. 